\title{
A HYGRO-THERMO-MECHANICAL CONSTITUTIVE MODEL FOR MULTIPHASE COMPOSITE MATERIALS
}

\author{
S. OLLER and E. OÑATE \\ Universitat Politècnica de Catalunya, E. T. S. Ingenieros de Caminos, Canales y Puertos, \\ Campus Nord U. P. C., Gran Capitán s/n, Modulo C1, 08034 Barcelona, Spain
}

\begin{abstract}
The paper describes a hygro-thermo-mechanical constitutive model appropriate for numerical simulation of multiphase composite material behaviour. The theory is based on the mixture of the basic substances of the composite and allows to evaluate the inter-dependent behaviour between the different compounding constitutive models. The initial anisotropy of each compounding can be taken into account by means of a mapped isotropic plastic formulation. This is a generalization of classic isotropic plasticity theory applied to orthotropic or anisotropic materials. The basic assumption is the existence of a stress and strain real anisotropic spaces and the respective fictitious isotropic spaces where a mapped fictitious problem is solved. Those spaces are related by means of two fourth order transformation tensor. The combination of mixing theory and the mapped isotropic plastic approach provides a powerful constitutive framework for the numerical treatment of bulk-fiber composite materials. Copyright (C) 1996 Elsevier Science Ltd.
\end{abstract}

\section{INTRODUCTION}

The analysis of composite material under thermal and hygrometric (moisture) loading is of great relevance in many practical problems in aerospace and automotive engineering. Thermal changes induce two important effects in a composite : standard volume changes of the form $E_{i j}^{\theta}=\alpha_{i j}^{\theta}\left(\theta-\theta_{o}\right)$ directly related with the thermal expansion tensor $\alpha_{i j}^{\theta}$ and the temperature increment $\left(\theta-\theta_{o}\right)$ (Malvern, 1969) and change in the mechanical properties of each point (Sluzalec, 1988), i.e., changes in the constitutive matrix and the yield strength of the material. Hygrometric loads induce an important effect in polimeric matrix components (Vinson et al., 1986). Dry polimeric matrices have good mechanical properties up to the vitreous transition temperature, above which the yield strength and the overall mechanical properties rapidly deteriorate. This critical temperature diminishes if moisture is retained within the matrix as a consequence of the combinations of high temperature and humidity leading to an increase in weight and swelling $(\leqslant 2 \%)$ as well as to the mentioned deterioration in the constitutive properties. This hygrometric effect is represented as $E_{i j}^{h}=\alpha_{i j}^{h}\left(h-h_{o}\right)$ where $\alpha_{i j}^{h}$ is the hygrometric expansion tensor and $\left(h-h_{o}\right)$ is the change of weight of the retained moisture with respect to the initial value $h_{o}$. It is clear that these two phenomena affect in a coupled manner the mechanical response of a composite. This paper attempts to model the constitutive behaviour of a composite material subjected to hygrothermal effects as well as external loads. It is therefore assumed that both the moisture and temperature values are known at each point of the composite. These values can be independently obtained by solving the corresponding hygrometric (Vinson et al., 1986) and thermal (Simo et al., 1991; Sluzac, 1988) problems.

The model proposed is based on the mixture of the basic substances in composites and allows the evaluation of the inter-dependence between the constitutive behaviour of the different compounding materials. The behaviour of the each compound is modelled by a general anisotropic hygro-thermal-elasto-plastic model, termed here "base model". The different base models for each compound are combined using mixing theory to simulate the behaviour of the multiphase bulk-fiber material.

Mixing theory was studied by Trusdell and Toupin (1960) and a few years later by Green and Naghdi (1965). Ortiz and Popov (1982) have used this theory to propose a two 
phase model for analysis of concrete. The authors have also used mixing theory to develop an implicit non linear constitutive model for $n$-phase composite materials. Each phase can have an anisotropic or isotropic behaviour defined by means of an equivalent isotropic solid. This idea was first introduced by Betten $(1981,1983,1988)$ using the concept of mapped stress tensor and later extended by Oller et al. (1993, 1993, 1995a, 1995b) to model the non linear constitutive behaviour of composites. The mapped stress concept offers the possibility of using all the merits of the well known isotropic models; consequently it has many computational advantages.

In this paper a generalization of standard isotropic theory to the analysis of anisotropic hygro-thermo-elasto-plastic solids is presented first. The anisotropic behaviour is formulated by means of fictitious isotropic stress and strain tensors which result from a fourth order tensor transformation of the real stress and strain fields to the homonymous stress and strain spaces. This allows the use of the same yield and potential functions derived for standard isotropic materials, whereas all the relevant information on the material anisotropy properties is embedded in the fourth order transformation tensors only. The material parameters involved in those tensors can be defined from adequate experimental tests. The extension of the base model to describe the orthotropic and anisotropic behaviour of composites using mixing theory is then detailed and the expressions of the secant and tangent constitutive operators are given. The model seems to be particularly suited to be applied for numerical analysis of multiphase materials such as fiber reinforced composites and concrete.

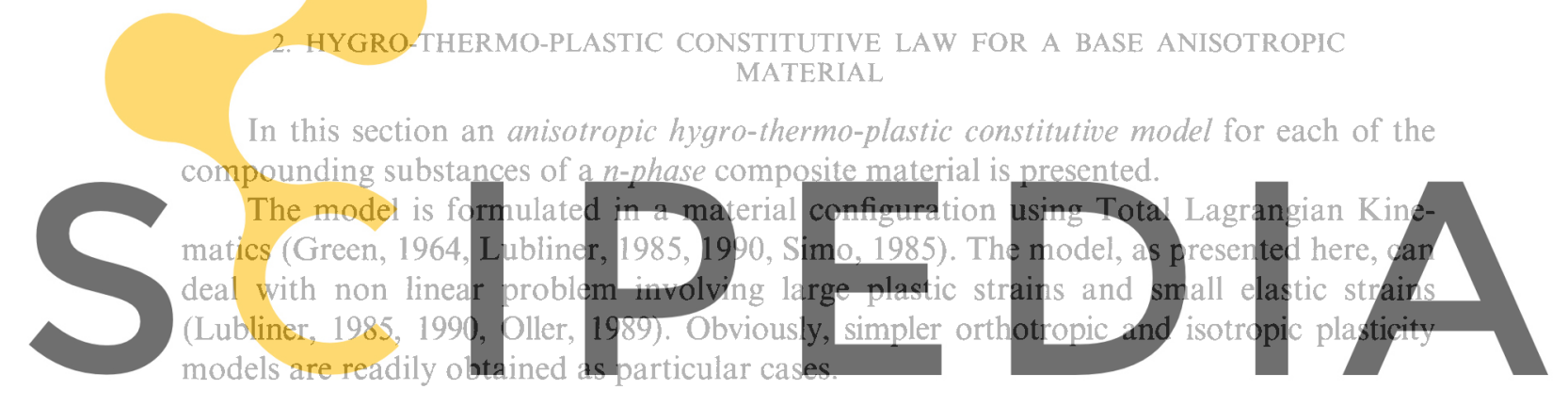

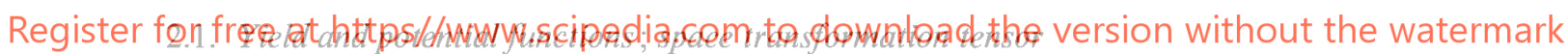

It will be assumed that both yield and plastic potential functions are defined in the Piola-Kirchoff stress space (material configuration) as:

$$
\mathscr{F}\left(S_{i j}, \theta, h, \not^{m}\right)=\mathscr{F}^{\tau}\left(\tau_{i j}, \theta, h, \not_{\tau}^{m}\right)=0, \quad \mathscr{G}\left(S_{i j}, \theta, h, \not^{m}\right)=\mathscr{G}^{\tau}\left(\tau_{i j}, \theta, h, \not_{\tau}^{m}\right)=\mathscr{K}
$$

where $S_{i j}$ and $\tau_{i j}$ are the second Piola-Kirchoff stress tensor in the real and mapped stress spaces, $\not^{m}$ and $\not_{\tau}^{m}$ are a set of $m$ internal plastic variables in both spaces, $\mathscr{K}$ is a constant parameter, $\theta$ is the temperature free variable and $h$ is the moisture measured in percentage weight increase.

Traditional procedures for deriving the constitutive equations for anisotropic elastoplastic materials are based on the description of appropriate yield and potential functions in terms of the characteristic material properties (Lubliner, 1990). Satisfaction of the invariance condition in these cases is difficult and not always possible (Malvern, 1969, Ziegler, 1983, Betten, 1981, 1988). A procedure to guarantee this condition is to define the properties of the real anisotropic solid in terms of a fictitious isotropic solid. This is achieved by relating the stresses between the real and fictitious spaces using the following linear transformation

$$
\tau_{i j}=A_{i j k l}^{\tau} S_{k l}
$$

where $S_{i j}$ and $\tau_{i j}$ are the stress tensor in the real anisotropic solid and the fictitious isotropic solid, respectively, and $A_{i j k l}^{\tau}$ is a fourth order material tensor, termed space transformation 
tensor influenced by the anisotropic strength properties. A possible definition of this tensor is the following:

$$
A_{i j k l}^{\tau}=f_{i k}^{\tau}(\theta, h) f_{j l}^{-1}(\theta, h)
$$

where $f_{i j}(\theta, h)$ and $f_{i j}^{\tau}(\theta, h)$ are the elastic strength limit tensors corresponding to the real and fictitious solids respectively. From hereafter superscript $\tau$ will denote material properties defined in the fictitious space.

To ensure no-proportionality between the strength and the elastic modulus the following relation between the real elastic strains $E_{i j}^{e}$ and the fictitious ones $\varepsilon_{i j}^{e}$ is defined

$$
\varepsilon_{i j}^{e}=A_{i j k l}^{\varepsilon} E_{k l}^{e}
$$

This assumption implies non-uniqueness of elastic strains when the change of space is produced. In eqn (4) $A_{i j k l}^{\varepsilon}$ is a fourth order material tensor, termed strain space transformation tensor influenced by the anisotropic stiffness properties. This can be derived from eqn (2), giving:

$$
A_{r s m n}^{\varepsilon}=\mathscr{C}_{r s i k}^{\tau-1} A_{i j k l}^{\tau} \mathscr{C}_{j l m n}, \quad \mathscr{C}_{j l m n}=A_{k l i j}^{\tau-1} \mathscr{C}_{i k t u}^{\tau} A_{\text {tumn }}^{\varepsilon}
$$

where $\mathscr{C}_{i j k l}(\theta, h)$ and $\mathscr{C}_{i j k l}^{\tau}(\theta, h)$ are the constitutive tensors in the real and fictitious spaces respectively, which are a function of the temperature $\theta$ and the moisture $h$.

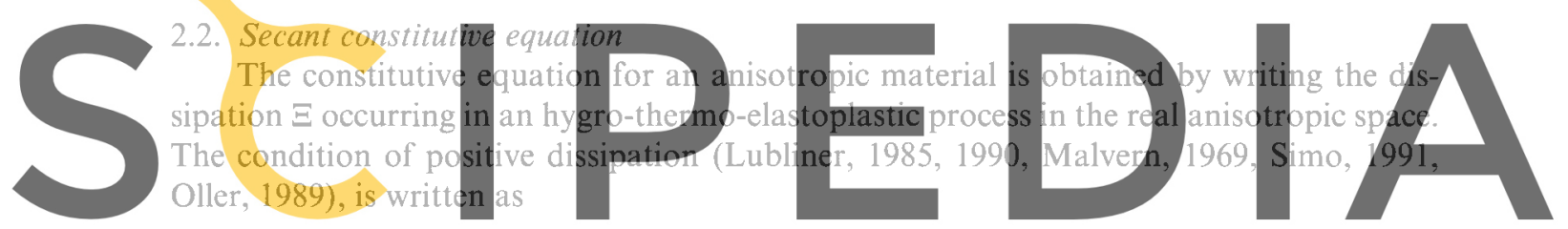

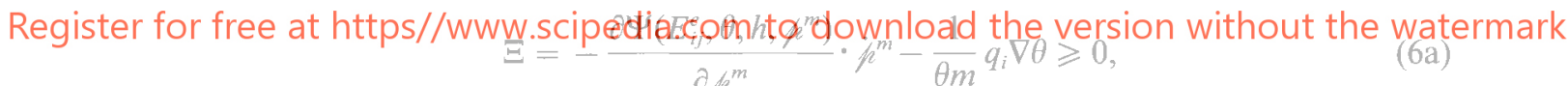

Using eqn (6a) the secant constitutive equation, for the hygro-thermo-mechanical problem, can be written as

$$
S_{i j}=m^{0} \frac{\partial \Psi\left(E_{i j}^{e}, \theta, h, h^{m}\right)}{\partial E_{i j}^{e}}=\mathscr{C}_{i j k l}(\theta, h) E_{k l}^{e}=A_{i j k l}^{\tau-1} \mathscr{C}_{k l r s}^{\tau}(\theta, h) \varepsilon_{r s}^{e}=A_{i j k l}^{\tau-1} \tau_{k l}
$$

and also the entropy $\eta$ as:

$$
\eta=-\frac{\partial \Psi\left(E_{i j}^{e}, \theta, h, \not^{m}\right)}{\partial \theta}=-\frac{1}{2 m_{o}} A_{i j k l}^{\varepsilon^{-1}} \varepsilon_{k l}^{e} \frac{\partial \mathscr{C}_{i j r s}^{\tau}(\theta, h)}{\partial \theta} \varepsilon_{r s}^{e}-\overbrace{\frac{\partial \Psi^{p}\left(\theta, h, \not^{m}\right)}{\partial \theta}}^{\eta^{p}}
$$

where $\Psi=\frac{1}{2} E_{i j}^{e} \mathscr{C}_{i j k l}(\theta, h) E_{k l}^{e}+\Psi^{p}$, and $\Psi^{p}$ are the total and plastic parts of free energy formulated in the material configuration under a real stress state (Oller et al., 1995) and $\eta^{p}$ is the plastic entropy (Armero et al., 1992). Note that the free energy can easily be formulated in the fictitious space in terms of the corresponding strain and constitutive tensors. Other thermomechanical constitutive variables can be derived from eqn (6b) and $(6 c)$, as : 


$$
\mathscr{C}_{i j k l}=m^{0} \frac{\partial^{2} \Psi\left(E_{p q}^{e}, \theta, h, \not \mu^{m}\right)}{\partial E_{i j}^{e} \partial E_{k l}^{e}}, \quad \mathscr{C}_{i j k l}^{\tau}=m^{0} \frac{\partial^{2} \Psi^{\rho}\left(\varepsilon_{p q}^{e}, \theta, h, \not \mu^{m}\right)}{\partial \varepsilon_{i j}^{e} \partial \varepsilon_{k l}^{e}}
$$

where $\mathscr{C}_{i j k l}$ and $\mathscr{C}_{i j k l}^{\tau}$ are the elastic constitutive tensors for the real and fictitious spaces, respectively.

$$
\begin{cases}\beta_{i j}^{\theta}=-m^{0} \frac{\partial^{2} \Psi\left(E_{p q}^{e}, \theta, h, \not^{m}\right)}{\partial E_{i j}^{e} \partial \theta} & \text { or } \quad \alpha_{i j}^{\theta}=-m^{0} \frac{\partial^{2} \Psi\left(E_{p q}^{e}, \theta, h, \not^{m}\right)}{\partial S_{i j} \partial \theta} \\ \beta_{i j}^{h}=-m^{0} \frac{\partial^{2} \Psi\left(E_{p q}^{e}, \theta, h, \not^{m}\right)}{\partial E_{i j}^{e} \partial h} & \text { or } \quad \alpha_{i j}^{h}=-m^{0} \frac{\partial^{2} \Psi\left(E_{p q}^{e}, \theta, h, \not^{m}\right)}{\partial S_{i j} \partial h}\end{cases}
$$

where $\alpha_{i j}^{\theta}$ and $\alpha_{i j}^{h}$ are the thermal and hygrometric expansion tensors, respectively, and $\beta_{i j}^{\theta}$ and $\beta_{i j}^{h}$ are the corresponding conjugate tensors. Note that all the tensors are defined in the real space. Their expressions in the fictitious space are given by

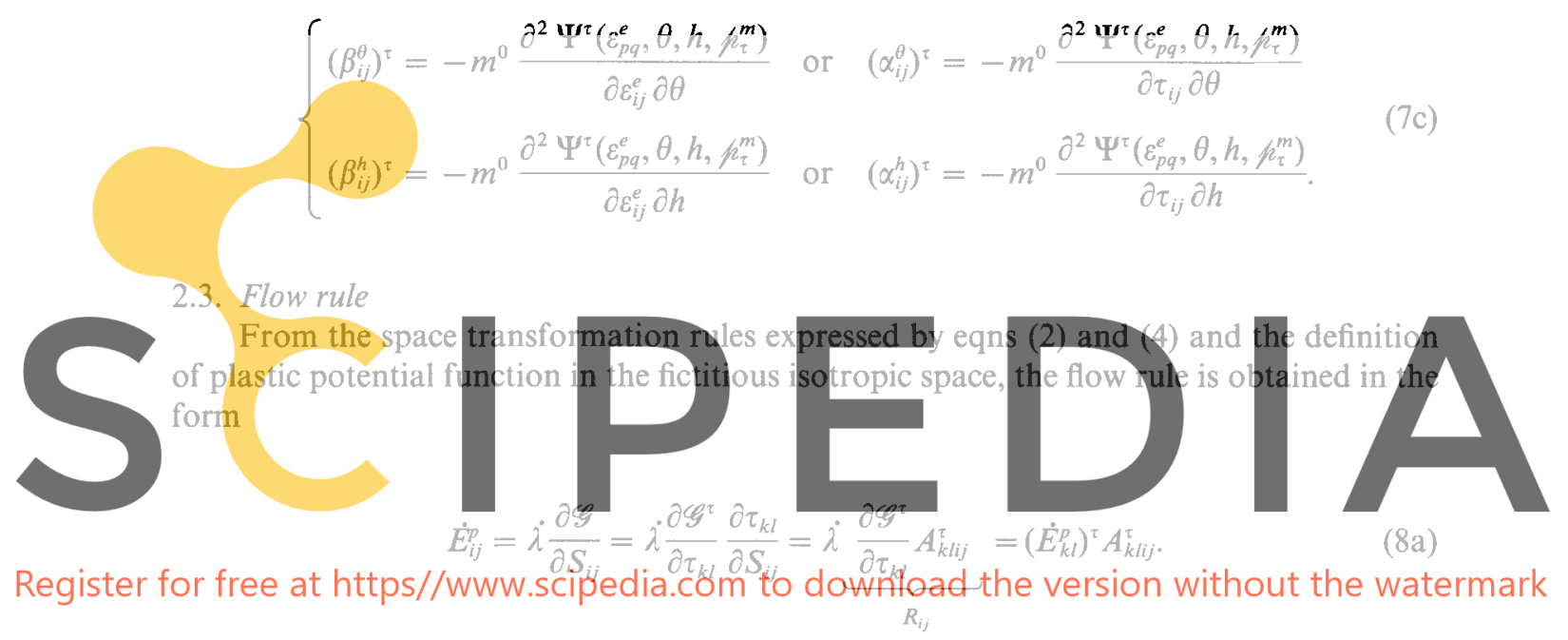

Combining eqns (8a) and (4) gives the plastic strain rates $\tilde{\varepsilon}_{i j}^{p}$ in the fictitious isotropic space as

$$
\dot{\varepsilon}_{r s}^{p}=A_{r s i j}^{\varepsilon} \dot{E}_{i j}^{p}=\underbrace{\dot{\lambda} A_{r s i j}^{\varepsilon} \underbrace{\overbrace{\frac{\partial G^{\tau}}{\partial \tau_{k l}}}^{R_{k l}^{\varepsilon}} A_{k l i j}^{\tau}}_{R_{i j}}}_{R_{r s,}}
$$

where $R_{k l}^{\tau}$ and $R_{i j}$ are respectively the plastic flows in the isotropic fictitious stress space and in the real stress space, influenced by the anisotropic strength ratio and $R_{r s *}$ is the plastic flow in the real stress space, taking into account all the stiffness and strength anisotropies.

\subsection{Tangent constitutive equation}

The rate form of the constitutive equation is obtained by performing the temporal derivatives of the secant expression (6b) as: 


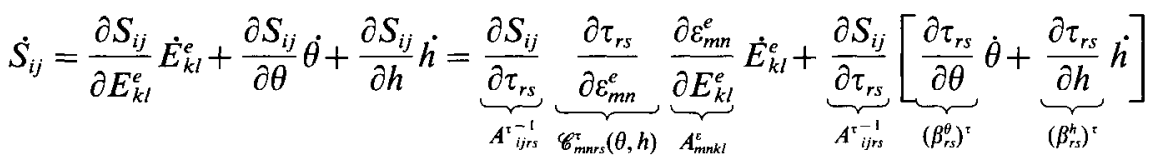

$$
\begin{aligned}
& =A_{i j r s}^{\tau-1} \underbrace{\mathscr{C}_{r s m n}^{\tau}(\theta, h) A_{m n k l}^{\varepsilon}\left(\dot{E}_{k l}-\dot{E}_{k l}^{p}\right)}_{t_{r s}}-A_{i j r s}^{\tau^{-1}}[\underbrace{\left(\beta_{r s}^{\theta}\right)^{\tau} \dot{\theta}}_{\dot{t}_{r s}^{\theta}=\mathscr{C}_{r s m m}^{r} \dot{\varepsilon}_{n m}^{\theta}}+\underbrace{\left(\beta_{r s}^{h}\right)^{\tau} \dot{h}}_{\dot{t}_{r s}^{h}=\mathscr{C}_{r s m m}^{\tau} \dot{\varepsilon}_{n m}^{h}}]
\end{aligned}
$$

The plastic consistency condition leads to the standard rate form of the constitutive equation in the fictitious isotropic space as:

$$
\begin{aligned}
& t_{i j}=\left\{\mathscr{C}_{i j k l}^{\tau}-\frac{\left(\mathscr{C}_{i j r s}^{\tau} R_{r s *}\right)\left(\frac{\partial \mathscr{F}^{\tau}}{\partial \tau_{r s}} \mathscr{C}_{r s k l}^{\tau}\right)}{-\Sigma_{m} \frac{\partial \mathscr{F}^{\tau}}{\partial \not_{\tau}^{m}}\left(H_{t u}^{m}\right)_{\tau} R_{t u}^{\tau}+\frac{\partial \mathscr{F}^{\tau}}{\partial \tau_{p q}} \mathscr{C}_{p q l n}^{\tau} R_{l n *}}\right\} \dot{\varepsilon}_{k l}
\end{aligned}
$$

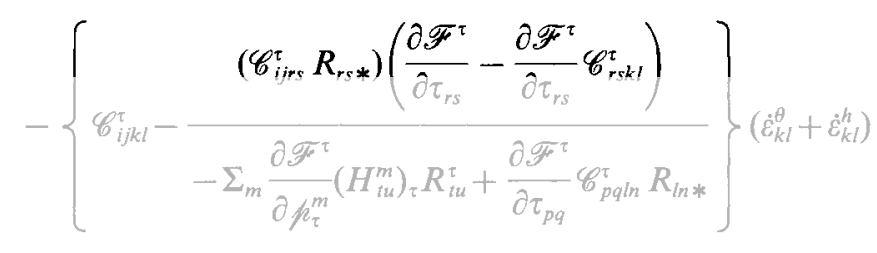

\section{or in compact form:}
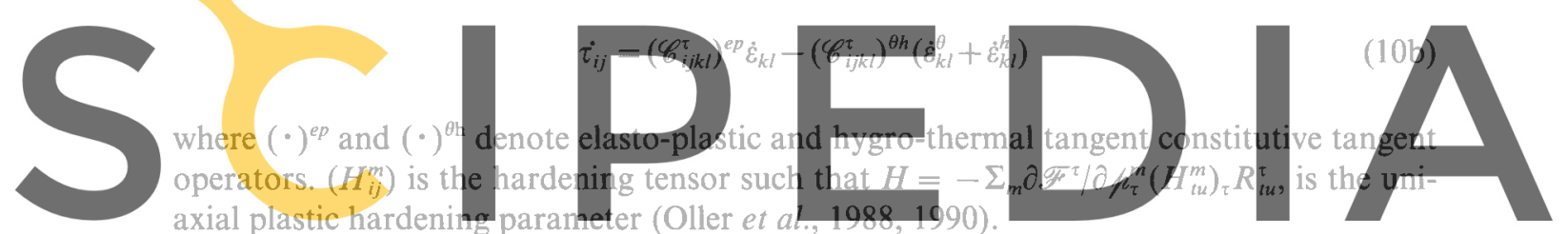

Substituting eqn (10) into (9) leads to the final expression of the rate constitutive

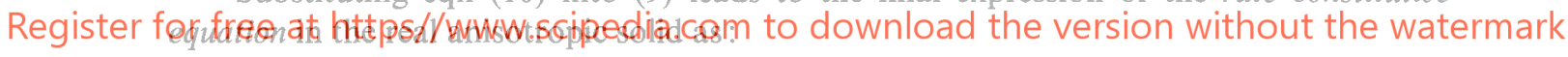

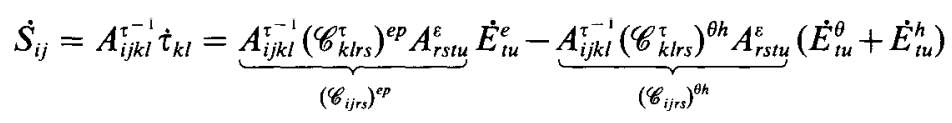

The above definitions allow to derive all the basic constitutive relationships and the evolution of the inner variables necessary to formulate the constitutive behaviour of a single anisotropic hygro-thermo-elasto-plastic solid (more details can be found in Oller et al., 1988, 1993, 1993, 1995, 1995, Luccioni, 1993).

\section{HYGRO-THERMO-MECHANICAL CONSTITUTIVE LAW FOR A MULTIPHASE COMPOSITE MATERIAL}

Mixing theory is based in the principle of interaction of the compounding substances and uses the following assumptions: (a) each infinitesimal volume of a composite is filled by a finite number of compounding substances; (b) each compounding substance participates in the behaviour of the total composite material in the same volume proportion of the total one; and (c) all compounding substances have the same strains (compatibility concept). Assumption (b) implies a homogeneous distribution of all substances in a certain region of the composite. The interaction between the different compounding substances, each one defined by an appropriate constitutive law, yields the overall constitutive behaviour of the composite in terms of the percentage volume occupied by each substance and its distribution within the composite. This theory allows to take into account the simultaneous 
combination of the different constitutive behaviours of each substance (i.e., elastic, elastoplastic, elasto-brittle elasto-damage, etc.). In this work it is assumed that each compounding substance behaves precisely as the anisotropic hygro-thermo-elasto-plastic material previously described. However, other constitutive combinations are obviously possible.

From hereafter notation $(\cdot)_{c}$ will denote the $c$ th material variable belonging to the single compounding previously defined.

The particular case of mixing theory used here is based on the assumption that, if the atomic diffusion is neglected (i.e., temperatures are moderate) the following strain compatibility condition at each point is satisfied:

$$
\left(E_{i j}\right)_{1}=\left(E_{i j}\right)_{2}=\cdots=\left(E_{i j}\right)_{c}=\cdots=\left(E_{i j}\right)_{n} \equiv E_{i j}
$$

Moreover, in composite materials the free energy can be written as (see Trusdell and Toupin, 1960)

$$
m^{0} \Psi\left(E_{i j}^{e}, \theta, h, \not^{m}\right)=m^{0} \Psi(E_{i j}, \theta, h, \underbrace{E_{i j}^{p}, \not^{m}}_{p^{m}})=m^{0} \sum_{c=1}^{n} k_{c} \Psi_{c}\left(E_{i j}, \theta, h,\left(p^{m}\right)_{c}\right)
$$

where $\Psi_{c}\left(E_{i j}, \theta, h,\left(p^{m}\right)_{c}\right)$ is the free energy corresponding to each compounding substance involved in the mixture, $k_{c}=\mathrm{d} V_{c} / \mathrm{d} V$ is the volume fraction of that substance, $\left(p^{m}\right)_{c}=$ $\left\{E_{i j}^{p}, h^{m}\right\}$ is a set of the inner variables for each compound, $\theta$ is the temperature acting as a free thermal variable and $h$ is the moisture measured in percentage weight increase.
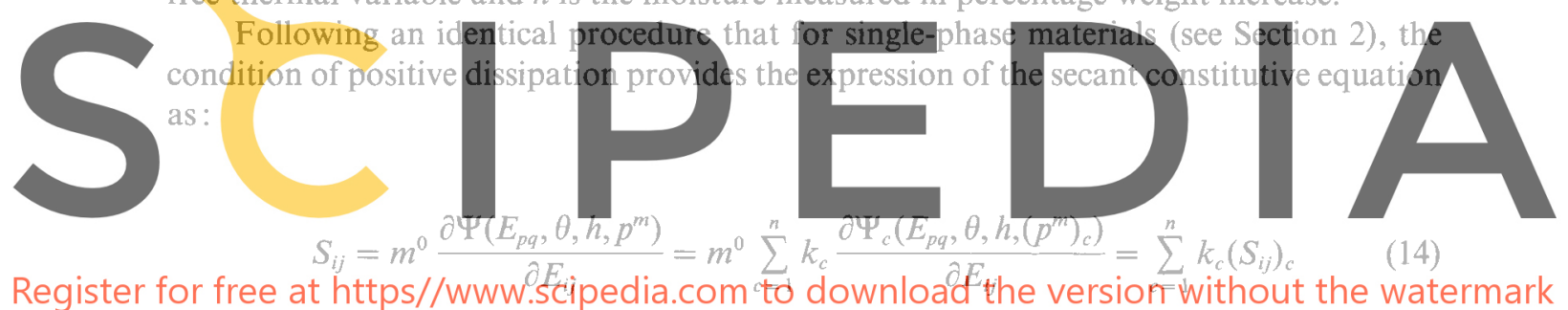

Register for free at https//www.Scipedia.com cto download the versiof w without the watermark

where $\left(S_{i j}\right)_{c}$ is the second Piola Kirchoff stress tensor for the $c$ th compounding substance.

Assuming strain additivity, i.e, $E_{i j}=E_{i j}^{e}+E_{i j}^{p}+E_{i j}^{\theta}+E_{i j}^{h}$, (Green-Naghdi, 1964), the stress-strain relationship is derived from the strain compatibility condition between substances (eqn (12)) as:

$$
\left(E_{i j}\right)_{c}=E_{i j}=(\underbrace{\left(\mathscr{C}_{i j k l}^{-1}(\theta, h)\right)_{c}\left(S_{k l}\right)_{c}}_{\left(E_{i j)}\right)_{c}}+\left(E_{i j}^{p}\right)_{c}+\underbrace{\left(\alpha_{i j}^{\theta}\right)_{c}\left(\theta-\theta_{o}\right)}_{\left(E_{i j}^{\theta}\right)_{c}}+\underbrace{\left(\alpha_{i j}^{h}\right)_{c}\left(h-h_{o}\right)}_{\left(E_{i j}^{h}\right)_{c}}
$$

where $\theta_{o}$ is the room temperature and $h_{o}$ is the referential moisture weight. The secant constitutive equation is obtained as:

$$
S_{i j}=\sum_{c=1}^{n} k_{c}\left(S_{i j}\right)_{c}=\sum_{c=1}^{n} k_{c}\left(\mathscr{C}_{i j k l}(\theta, h)\right)_{c}\left(E_{k l}^{e}\right)_{c}=\mathscr{C}_{i j k l}(\theta, h) E_{k l}^{e}
$$

where the elastic strain for each compound can be expressed as: $\left(E_{i j}^{e}\right)_{c}=E_{i j}-\left(E_{i j}^{p}\right)_{c}-$ $\left(\alpha_{i j}^{\theta}\right)_{c}\left(\theta-\theta_{o}\right)-\left(\alpha_{i j}^{h}\right)_{c}\left(h-h_{o}\right)$, and for the composite as: $E_{i j}^{e}=E_{i j}-E_{i j}^{p}-\alpha_{i j}^{\theta}\left(\theta-\theta_{o}\right)-$ $\alpha_{i j}^{h}\left(h-h_{0}\right)$. From the last two members of eqn (16) it is possible to write the total plastic strain for the composite material as: 


$$
\begin{aligned}
E_{i j}^{p}=\mathscr{C}_{i j p q}^{-1}(\theta, h)\left\{\sum_{c=1}^{n} k_{c}\left(\mathscr{C}_{p q r s}(\theta, h)\right)_{c}\left[\left(E_{r s}^{p}\right)_{c}+\left(\alpha_{r s}^{\theta}\right)_{c}\left(\theta-\theta_{o}\right)+\left(\alpha_{r s}^{h}\right)_{c}\left(h-h_{o}\right)\right]\right\} & \\
& -\left[\alpha_{i j}^{\theta}\left(\theta-\theta_{o}\right)+\alpha_{r s}^{h}\left(h-h_{o}\right)\right]
\end{aligned}
$$

where the plastic strain for the $c$ th compound is:

$$
\left(E_{i j}^{p}\right)_{c}=\int_{o}^{t}\left(\dot{E}_{i j}^{p}\right)_{c} \mathrm{~d} t
$$

and the plastic strain rate was defined in eqn (8). From the last equation it is possible to obtain the stress tensor for each phase as:

$$
\begin{array}{r}
\left(S_{i j}\right)_{c}=\left(\mathscr{C}_{i j p q}(\theta, h)\right)_{c}\left\{\mathscr{C}_{p q k i}^{-1}(\theta, h)\left[\sum_{c=1}^{n} k_{c}\left(\mathscr{C}_{k l r s}(\theta, h)\right)_{c} E_{r s}\right]\right. \\
\left.-\left(E_{p q}^{p}\right)_{c}-\left(\alpha_{p q}^{\theta}\right)_{c}\left(\theta-\theta_{o}\right)-\left(\alpha_{p q}^{h}\right)_{c}\left(h-h_{o}\right)\right\}
\end{array}
$$

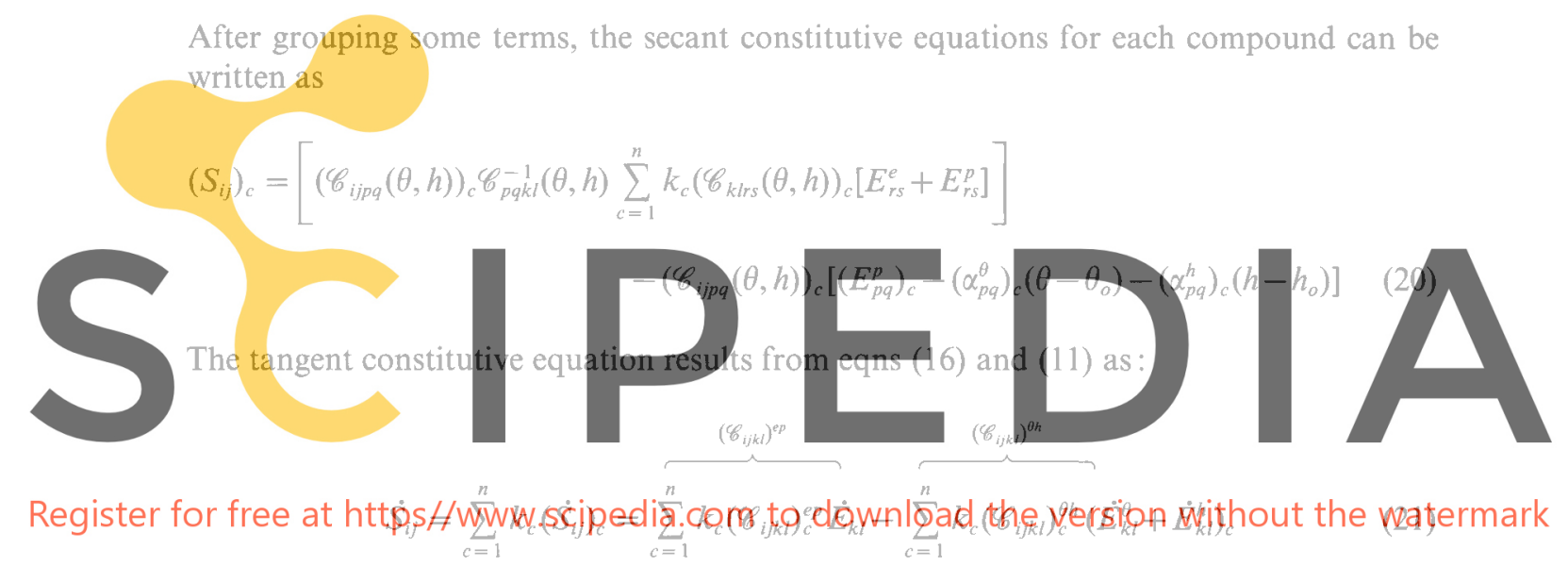

where the expression for the tangent operators $\left(\mathscr{C}_{i j k l}\right)_{c}^{e p},\left(\mathscr{C}_{i j k l}\right)_{c}^{\theta h}$ can be found in eqn (11). Equation (20) gives the hygro-thermo-elasto-plastic stress for each compounding substance of the multiphase composite and eqn (21) its rate.

\section{CONCLUDING REMARKS}

The theoretical framework presented here combines basic concepts from multiphase mixing theory with an anisotropic mapped formulation. Such framework provides a powerful tool for modeling the hygro-thermo-mechanic constitutive behaviour of composite materials. This model can be used for the numerical analysis of fracture behaviour of fiber reinforced composites and reinforced concrete structures as shown in Oller et al. (1995a, 1995b).

\section{REFERENCES}

Armero, F. and Simo, J. (1992). Product formula algorithms for non linear coupled thermoplasticity: formulation and non linear stability analysis. SUDAM Report No 92-94, Stanford University.

Betten, J. (1981). Creep theory of anisotropic solids. J. Rheology 25, 565-581.

Betten, J. (1983). Damage tensor in continuum mechanics. J. Mècanique Théorique et Appliqué 2, 13-32.

Betten, J. (1988). Application of tensor functions to the formulation of yield criteria for anisotropic materials. Int. J. Plasticity 4, 29-46.

Green, A. and Naghdi, P. (1964). A general theory of an elastic-plastic continuum. Arch. Rational Mech. Anal. 18, 19-281.

Green, A. and Naghdi, P. (1965). A dynamical theory of interacting continua. Int. J. Engng Sci. 3, 231. 
Lubliner, J. (1972). On thermodynamics foundations of non-linear solid mechanics. Int. J. Non-linear Mechanics 7, 237-254.

Lubliner, J. (1985). Thermomechanics of Deformable Bodies. Department of Civil Engineering, University of California, Berkeley, U.S.A.

Lubliner, J. (1986). Normality rules in large-deformation plasticity. Mechanics Mat. 5, 29-34.

Lubliner, J., Oliver, J., Oller, S. and Oñate, E. (1989). A plastic damage model for non linear analysis of concrete. Int. J. Solids Structures 25, 299-326.

Lubliner, J. (1990). Plasticity Theory. Macmillan Publishing, U.S.A.

Luccioni, B. (1993). Formulación de un modelo constitutivo para materiales ortótropos. PhD. thesis, Universidad de Tucamán, Argentina.

Malvern, L. (1969). Introduction to the Mechanics of Continuous Medium. Prentice Hall, U.S.A

Oliver, J., Cervera, M., Oller, S. and Lubliner, J. (1990). Isotropic damage models and smeared crack analysis of concrete. In SCI-C1990, Second Int. Conf. on Computer Aided Design of Concrete Structure, Zell am See, Austria, pp. 945-957.

Oller, S. (1988). Un Modelo de daño continuo para materiales friccionales. PhD thesis, Escuela Tecnica Superior de Ingenieros de Caminos Canales y Puertos de Barcelona.

Oller, S. (1989). Nuevos Materiales Estructurales, Cerámicos en Ingenieria. Centro Internacional de Métodos Numéricos en Ingenieria, Barcelona, España.

Oller, S., Oñate, E., Oliver, J. and Lubliner, J. (1990). Finite element non-linear analysis of concrete structures using a plastic-damage model. Engng Fract. Mech. 35, 219-231.

Oller, S., Oñate, E., Miquel, J. and Botello, S. (1993). A finite model for analysis of multiphase composite materials. Ninth Int. Conf. Composite Materials. Madrid, Spain.

Oller, S., Oñate, E. and Miquel, J. (1993). Simulation of anisotropic elastic-plastic behaviour of materials by means of an isotropic formulation. 2nd U.S. Nat. Congress on Computational Mechanics, Washington, U.S.A.

Oller, S., Oñate, E., Miquel, J. and Botello, S. (1995 a). A plastic damage constitutive model for composite materials. Int. J. Solids Structures (submitted).

Oller, S., Botello, S., Miquel, J. and Oñate, E. (1995 b). An anisotropic elasto-plastic model based on an isotropic formulation. Int. J. Engng Computation 12, 245-262.

Oñate, E., Oller, S., Botello, S. and Miquel, J. (1991). Metodos avanzadoes de calculo de estructuras de materiales compuestos. Ed. C.I.M.N.E., Nro. 11, Barcelona, Spain.

Ortiz, M. and Popov, E. P. (1982). Plain concrete as a composite material. Mech. Mat. 1, 139-150.

Simo, J. (1985). On the computational significance of the intermediate configuration and hyperelastic stress relations in finite deformation elastoplasticity. Mech. Mat. 4, 439-451.

Simo, J. and Hugges, T. (1991). Plasticity, Viscoplasticity and Viscoelasticity: Formulation and Numerical Analysis

Aspects. Springer-Verlag, Berlin,
Simo, J. and Ju, J. (1987). Strain Struchures 23, 821-840.

Sluzalec, A. (1988). An analysis of ther Appl. Num. Meth. 4, 67

Trusdell, C. and Toupin, Verlag, Berlin.

Vinson, J. and Sierakowski, R. (1986). The Behaviour of Structures Composed of Composite Materials. Martinus Nijhoff Publishers. 